\title{
The speed of light or the speeds of light?
}

\author{
Elmo Benedetto $^{* 1,2 @}$, Gerardo Iovane ${ }^{1,2}$ \\ ${ }^{1}$ University of Salerno, Department of Computer Science, Fisciano, Italy. \\ ${ }^{2}$ High School S. Caterina da Siena-Amendola, Salerno, Italy.
}

Received on December 02, 2021. Revised on January 12, 2022. Accepted on January 31, 2022.

\begin{abstract}
The Italian government's Ministry of Public Education, which defines the high school curricula, has introduced almost all the topics of modern physics for students in their last year of high school. The theory of relativity is, perhaps, the argument most developed. The students, doing a web search, read that the speed of light can take different values in General Relativity (GR). We have noticed that this concept creates a lot of confusion and also a misconception in young students. In this paper, we want to describe a lecture given in the classroom about this topic.
\end{abstract}

Keywords: Speed of light, Gravitational time dilation, General relativity.

\section{Introduction}

The theory of relativity is now being studied at a high school level [1]. However, many things that are obvious for graduates are not obvious to younger students. For this reason, many topics require didactic insights $[7-13]$. The starting point of GR, in addition to the equivalence principle, is the so-called principle of general covariance [14. The physical laws can be written in a form that does not depend on the coordinate system. Coordinates are only numbers that give us a one-to-one correspondence between events and $\mathbb{R}^{4}$ where $\mathbb{R}^{4}$ is the set which consists of all $(x, y, z, t)$ with $x, y$, $z, t$ real numbers. Pupils know that to calculate the speed of a body they must divide space by time. The problem is that the time flows differently from point to point and the difference in spatial coordinates may not coincide with the physical distance. Even if the difference between the spatial coordinates coincides with the physical distance, what time should we use? We have an infinite number of clocks and, therefore, an infinite number of different speeds. In GR, the velocity that corresponds to the concept of velocity in classical physics and special relativity is the 'local velocity', which is the velocity of a body in an infinitesimal region of spacetime, as measured by an observer who is there. In class we have named it "real velocity". The real speed of light is always equal to $c$ also in GR. In other words, while in classical physics and special relativity the velocity is a global concept, in GR it becomes a local concept. Therefore defining the velocity of distant bodies could become ambiguous. For this reason, we have never dealt with this topic in high school. However, students can read online, that in GR, the speed of light might have

\footnotetext{
${ }^{*}$ Correspondence email address: elmobenedetto@libero.it
}

values other than $c$ and this could lead them to a wrong interpretation. We have observed that even the most talented pupils believe that really light slows down or accelerates. So we have decided to give a lesson in class about this subject, by analyzing the speed of free fall. This motion is analyzed by a local observer and by an observer outside the gravitational field.

\section{Free Fall}

Using the principle of energy conservation, students know how to calculate the rate of fall of a test body in a gravitational field of a massive body getting

$$
v_{\tau}=\sqrt{\frac{2 G M}{R}}
$$

where $R$ is the distance from the center of the mass $M$ that generates the gravitational field. If the body starts from a standstill and from far away, it reaches the speed of light when $R=R_{s}=\frac{2 G M}{c^{2}}$ where $R_{s}$ is the so called Schwarzschild radius of black hole. The speed always increases during the fall and reaches its maximum value when the distance from the center has the value just written. Against which clock is this speed calculated? It is the real speed and is calculated using the clocks that are at rest in the gravitational field at the points where the body passes. What is the link between the time $\tau$ measured by these clocks and the time $t$ measured by clocks outside the gravitational field, i.e., for an observer at an arbitrarily large distance from $M$ ? In every university textbook this link is demonstrated but they have simply taken it from the wikipedia page [15]

$$
\tau=t \sqrt{1-\frac{2 G M}{c^{2} R}}
$$

For this reason, we can calculate the rate of change of $R$ with respect to $t$, and that is the "apparent velocity", 
as measured by the observer outside the gravitational field

$$
v_{t}=\frac{d R}{d t}=\frac{d R}{d \tau} \frac{d \tau}{d t}=v_{\tau} \sqrt{1-\frac{2 G M}{c^{2} R}} .
$$

Therefore

$$
v_{t}=\sqrt{\frac{2 G M}{R}} \cdot \sqrt{1-\frac{2 G M}{c^{2} R}}=\sqrt{\frac{2 G M}{R}-\frac{4 G^{2} M^{2}}{c^{2} R^{2}}} .
$$

The domain of the previous function is $\left[R_{s},+\infty\right]$. Obviously, the distance $R$ is the Schwarzschild radial coordinate but we neglected this by telling students to consider it exactly as spatial distance. The derivative with respect to $R$ is

$$
\frac{d v_{t}}{d R}=\frac{-2 G M c^{4} R^{2}+8 G^{2} M^{2} c^{2} R}{2 c^{4} R^{4} \sqrt{\frac{2 G M}{R}-\frac{4 G^{2} M^{2}}{c^{2} R^{2}}}},
$$

and we deduce that the maximum value is $c / 2$ and occurs at $R=\frac{4 G M}{c^{2}}$ and, that is, the coordinates of the maximum are

$$
\left(\frac{4 G M}{c^{2}}, \frac{c}{2}\right)
$$

Moreover, we have

$$
\left\{\begin{array}{l}
\lim _{R \rightarrow+\infty} v_{t}=0 \\
\lim _{R \rightarrow R_{s}} v_{t}=0
\end{array}\right.
$$

We saw that the real velocity, $v_{\tau}$, started as zero, at $R=\infty$, and increases to a maximum of $c$, at $R=R_{s}$. The apparent velocity, $v_{t}$, has a totally different behavior. It starts from zero, increases to half the speed of light, then decreases back to zero.

\section{Speed of Light}

If now we consider the apparent velocity of a photon, $v_{c}$, we get

$$
v_{c}=\frac{d R}{d t}=\frac{d R}{d \tau} \frac{d \tau}{d t}=c \sqrt{1-\frac{2 G M}{c^{2} R}} .
$$

The function $v_{c}=f(R)$ is always increasing

$$
\left\{\begin{array}{l}
\lim _{R \rightarrow+\infty} v_{c}=c \\
\lim _{R \rightarrow R_{s}} v_{c}=0
\end{array}\right.
$$

Therefore, for the external observer, the photon starts at the speed of light and begins to decelerate. Finally it stops on the Event Horizon. Therefore, the apparent speed of light slows down.

\section{Circular Motion}

At this point we finished the lesson but the students proposed to analyze the case of circular orbits. In fact, they found the gravitational time dilation from the same wikipedia page

$$
\tau=t \sqrt{1-\frac{3 G M}{c^{2} R}}=t \sqrt{1-\frac{3 R_{s}}{2 R}},
$$

where $\frac{3}{2} R_{s}$ is the radius of the so called photon sphere, and the following correction to the the Newtonian circular velocity 16

$$
v_{\tau}=\sqrt{\frac{G M}{R-R_{s}}} .
$$

If we pose $v_{\tau}=c$, it is easy to see that a photon is in orbit around the mass when $R=\frac{3}{2} R_{s}$. In this case, from (10) we have

$$
v_{c}=\frac{d(R \cdot \theta)}{d t}=\frac{d(R \cdot \theta)}{d \tau} \frac{d \tau}{d t}=c \sqrt{1-\frac{3 G M}{c^{2} R}}=0 .
$$

So the photon appears to not revolve around the mass as if it were frozen. We want to point out that the definition of velocities in cosmology seems to be much more puzzling at high school level. The concept of distance and velocity in an expanding universe was addressed in a previous paper [17].

\section{Conclusion}

In this paper, we have summarized a lecture given in a high school class (students 17-18 years). The students were very fascinated by Einstein's theory and we have tried to analyze a not very simple concept for their young age. Indeed, we discussed the concept of velocity in general relativity. We first considered a massive body in free fall in a gravitational field by noting how, an observer at an arbitrarily large distance from the source of gravity, measures a rate of fall totally different from the real velocity of free fall. This allowed us to introduce some concepts, such as the black hole, which, has, as expected, stimulated the interest of the pupils. We then focused on the speed of light and considered both radial and circular motion. Using the mathematical formalism appropriate for their age, we tried to understand how, as a consequence of the time dilation, even the apparent speed of light is not constant and in some cases the light can even seem frozen.

\section{Acknowledgements}

This research was partially supported by FAR fund of the University of Salerno. 


\section{References}

[1] I.M. Egdall, The Physics Teacher 47, 522 (2009).

[2] I.M. Egdall, The Physics Teacher 52, 406 (2014).

[3] E.S. Ginsberg, The Physics Teacher 58, 373 (2020).

[4] D.V. Redžić, European Journal of Physics 29, 191 (2008).

[5] W.B. Stannard, Physics Education 53, 035013 (2018).

[6] M.S. Alvarez-Alvarado, C. Mora and C.B. CevallosReyes, Revista Brasileira de Ensino de Física 41, e20190008 (2019).

[7] E. Benedetto, Afrika Matematika 28, 23 (2017).

[8] A. Feoli, A.L. Iannella and E. Benedetto, Revista Brasileira de Ensino de Física 43, e20210017 (2021).

[9] E. Benedetto, A. Briscione and G. Iovane, Revista Brasileira de Ensino de Física 43, e20200515 (2021).

[10] E. Benedetto and A. Feoli, European Journal of Physics 38, 055601 (2017).

[11] A. Feoli and E. Benedetto, Physics Education 56, 015002 (2021).

[12] D.V. Redžić, European Journal of Physics 39, 025205 (2018).

[13] E. Hecht, The Physics Teacher 59, 27 (2021).

[14] P.G. Bergmann, in: Introduction to the Theory of Relativity (Dover, New York, 1976).

[15] WIKIPEDIA, Gravitational time dilation, available in: https://en.wikipedia.org/wiki/Gravitational_time_dil ation accessed in 20/01/2020.

[16] C. Miller, Equation of Motion and Geodesics, available in: https://www.astro.umd.edu/ miller/teaching/astr4 98/lecture10.pdf, accessed in 20/01/2020.

[17] E. Benedetto, G. Iovane, B. Sersante and F. Feleppa, Canadian Journal of Physics 98, 7 (2020). 